\section{Panacéias disseminadas pela Internet e pacientes vulneráveis: como conter um mercado de ilusões?}

\author{
Paulo R. Vasconcellos-Silva, ${ }^{1}$ \\ Luis David Castiel, ${ }^{2}$ \\ Marcos Bagrichevsky ${ }^{3}$ \\ e Rosane Harter Griep 4
}

Como citar: Vasconcellos-Silva PR, Castiel LD, Bagrichevsky M, Griep RH. Panacéias disseminadas pela Internet e pacientes vulneráveis: como conter um mercado de ilusões? Rev Panam Salud Publica. 2011;29(6):469-74.

\begin{abstract}
SINOPSE
O presente artigo discute a proliferação de curas e terapias fraudulentas que surgem e evanescem cotidianamente na Internet. A clientela dessas fraudes, vulnerabilizada pela doença e pela perspectiva da morte, utiliza a Internet para adquirir produtos que seriam possivelmente ignorados em outros contextos. Tal vulnerabilidade se liga a contingências físicas desgastantes que comprometem a capacidade de decisão. A tentativa de controlar o fenômeno das fraudes de forma estritamente racional, sem levar em conta a vulnerabilidade desse consumidor que pouco tem a perder e sem considerar suas demandas de integralidade, pode conduzir a resultados desapontadores, já que as panacéias parecem estar preenchendo os lapsos deixados por estruturas assistenciais que têm sido insensíveis à imaterialidade dos medos humanos.
\end{abstract}

Palavras-chave: Internet; panacéia; charlatanismo; vulnerabilidade em saúde; comunicação em saúde.

\footnotetext{
Fundação Oswaldo Cruz, Instituto Oswaldo Cruz, Laboratório de Inovações em Terapias, Ensino e Bioprodutos (LITEB), Rio de Janeiro (RJ), Brasil. Correspondência: bioeticaunirio@yahoo.com.br

2 Fundação Oswaldo Cruz, Escola Nacional de Saúde Pública Sergio Arouca (ENSP), Rio de Janeiro (RJ), Brasil.

3 Universidade Federal do Espírito Santo (UFES), Programa de PósGraduação em Educação Física, Vitória (ES), Brasil.

4 Fundação Oswaldo Cruz, Instituto Oswaldo Cruz, Laboratório de Educação em Ambiente e Saúde (LEAS), Rio de Janeiro (RJ), Brasil.
}

Existe na Internet uma demanda crescente pela comercialização de métodos terapêuticos fraudulentos que, não raro, os sistemas jurídicos hesitam em enquadrar e as agências de regulação fracassam em reprimir. As pesquisas no terreno são, ainda, dificultadas pela agilidade com que atualmente é possível publicar um site e retirá-lo da Internet. Infelizmente, graças aos muitos recursos de acesso peculiares a essa rede, há condições ideais para a divulgação desse tipo de atividade, já categorizada há décadas por alguns como grave problema de saúde pública (1).

As estatísticas acerca da venda de tratamentos fraudulentos pela Internet são ainda escassas, em decorrência da complexidade envolvida na localização daqueles que querem se ocultar de sistemas de vigilância, o que também dificulta a elaboração e a aplicação de metodologia adequada ao estudo do problema. Há autores que associam o aumento do consumo ao excesso da oferta (2), o que poderia ser, de alguma forma, contornado pela regulamentação e fiscalização da Internet - uma medida que se chocaria com os princípios fundadores e norteadores dessa grande rede. Assim, em virtude dos seus atributos de acessibilidade, instantaneidade e fugacidade (3), a Internet é uma vitrine ideal para esse tipo de comércio, colocado à disposição de potenciais clientes, sobretudo dos que buscam recursos que os aliviem de condições de padecimento crônico ou da iminência de uma morte dolorosa. Além disso, as inclinações sensacionalistas e a escassez de visão crítica no campo do jornalismo científico que fazem dispensar a checagem junto a fontes qualificadas - não raro favorecem a divulgação de conteúdos (4) que se valem da boa-fé ou de angústias mal atendidas pela medicina. De outro lado, as agências regulamentadoras produzem advertências e apelos enfáticos, com tentativas recorrentes de intervir em um mercado de ilusões que tem a oferecer produtos elaborados na medida das expectativas dos incautos, não raro vulnerabilizados por dores e anseios frustrados pelos métodos convencionais.

A Internet parece ter se tornado, como afirma Ross, uma terra sem lei (5), em vista do crescente interesse e acesso facilitado aos textos técnicos que se misturam aos sites de charlatões. Estes encontram uma clientela potencial entre pacientes que apresentam sua capacidade de julgamento comprometida por condições crônicas desgastantes e doenças com prognóstico sombrio. A privação temporária da capacidade de julgamento dessa clientela parece não se relacionar a fatores educacionais, assim como o crescimento da oferta desses terapeutas não parece se dever à escassez de selos de qualidade e códigos de conduta entre os sites sobre saúde. Desde a invenção da prensa móvel, o acesso a informações nunca foi tão facilitado, tanto 
para aqueles que se instruem com material produzido por profissionais qualificados, como para os que buscam por esperanças de qualquer origem.

O objetivo deste artigo é analisar esse fenômeno cultural em expansão, considerando a vulnerabilidade exacerbada pelas condições crônicas e a temporária privação de discernimento originada no sofrimento, no contexto da facilidade de acesso à Internet, com sua mistura indiscriminada de informações.

\section{CARACTERÍSTICAS DA FRAUDE E VIGILANTES DO COMÉRCIO FRAUDULENTO}

Uma das características historicamente mais estáveis dos fraudadores é a promoção de produtos em formatos que se assemelhem a informações técnicas qualificadas $(6,7)$, como já foi descrito nos trabalhos historiográficos de Porter $(8,9)$ sobre o charlatanismo da emergente sociedade liberal inglesa. Nos Estados Unidos, a Food and Drug Administration (FDA) define como fraude em saúde - quackery - a promoção de tratamentos falsos ou de eficácia sabidamente não comprovada com vistas ao lucro (1). Isso difere em quase todos os aspectos dos sistemas religiosos de cura, fenômeno cultural suficientemente investigado pela antropologia cultural e até mesmo pela epidemiologia social. Observa-se um crescimento em ritmo exponencial no número de sites que divulgam tratamentos genericamente conhecidos como medicina alternativa (so-called alternative medicine, SCAM) e que se valem de sistemas e perspectivas metafísicas à procura de ingênuos e vulneráveis (10). Por meio desses sites, charlatões convencem consumidores acerca das habilidades de cura de produtos miraculosos (11).

As preocupações quanto a segurança e fiabilidade das informações sobre saúde disponibilizadas na web a tornaram prolífica em códigos de conduta e sistemas de avaliação de qualidade (12). A fundação Health on the Net (HON) destaca oito princípios éticos em seu código de conduta (13) - princípios aos quais os sites fraudulentos não têm como aderir, justamente porque querem confundir o olhar leigo. Contudo, há extensas áreas cinzentas, nas quais o embate entre perspectivas terapêuticas se prolonga até o terreno jurídico. É emblemático o caso de Starchild Abraham Cherrix, um jovem norte-americano com doença de Hodgkin que optou, juntamente com a família, por se tratar com uma dieta de suplementos alimentares orgânicos e isenta de açúcar ao invés de submeter-se à quimioterapia. A justiça do Estado da Virgínia determinou que os pais encaminhassem o jovem a um serviço de oncologia clínica sob pena de perda da guarda, o que não impediu que o casal Cherrix recorresse a instâncias superiores $(14,15)$.

Em vista da progressão alarmante de fraudes divulgadas pela $w e b$, diversas agências norte-americanas se uniram ao redor do que foi chamado de Operation Cure.All - uma alusão aos sites que prometiam a cura para todos os males $(16,17)$. Segundo os relatórios desse grupo, as seguintes características seriam sinais de perigo: produtos anunciados como naturais ou não tóxi- cos, sugerindo a ausência de efeitos colaterais; testemunho de clientes satisfeitos com curas prodigiosas; ingredientes secretos, usados há milênios ou que encerrem descobertas científicas fenomenais ainda não reconhecidas pela medicina; cura de uma grande variedade de males desconexos; uso indiscriminado de termos médicos; exclusividade na comercialização, assim como necessidade de pagamento adiantado e garantia de ressarcimento das despesas no caso de insucessos; sites sem divulgação de endereços físicos, números de telefone ou outra forma de contato (16) e, previsivelmente, sem selos de qualidade como o da HON.

Em 2001, o problema foi considerado, nos Estados Unidos, como um grave caso de saúde pública pela Comissão Federal de Comércio. Essa comissão lançou então uma espécie de "cyber cruzada" contra os comerciantes de panacéias na Internet (17). Artigos publicados em outros continentes dão conta da dimensão global do problema (18-20). A Comissão Federal de Comércio relata casos de pacientes com câncer que cancelaram cirurgias, quimioterapia ou radioterapia, vulneráveis às promessas de cura por meio de ervas que podem custar centenas de dólares e abrindo mão, possivelmente, de suas últimas oportunidades de intervenção sobre a doença $(17,21)$. Ao contrário das antigas panacéias e "óleos de cobra" anunciados amplamente nas grandes cidades $(8,9,22)$, os sites fraudulentos oferecem três dificuldades à sua identificação e regulamentação: são de autoria falsa, evanescentes e globalizados (23).

No rastro dos fraudadores, além das instituições reguladoras e dos códigos de qualidade para o consumo de informações em saúde, é frequente encontrar outro tipo de vigilante. Existe um número crescente de organizações de defesa do consumidor em saúde na Internet (geralmente patrocinadas por sociedades médicas), organizações não governamentais e sites pessoais $(24,25)$ que acreditam na suficiência das estratégias racionalistas, centradas nas evidências mais atualizadas e em informações qualificadas, para refrear o assédio aos emocionalmente vulneráveis. Ocupam-se do rastreamento de práticas de eficácia não comprovada (ou explícito charlatanismo). O panorama apontado por esses portais permite uma familiarização com a lógica de marketing das terapias viciosas (tabela 1).

A história dos vigilantes das fraudes parece tão antiga quanto a dos tratamentos viciosos. Huisman (26) ilustra a história das panacéias com dados históricos acerca da luta dos "educadores do povo" contra os fraudadores holandeses no século XIX, produzindo um retrato atual acerca do embate de credibilidade entre a fraude e as luzes da ciência. Assim como os educadores de hoje, os irmãos holandeses retratavam o fenômeno como um caso de ignorância social, que assolava não somente as classes desfavorecidas, mas também (como se observa contemporaneamente) os mais ricos e instruídos. Outra similitude é a convicção de que somente o discurso médico-científico deveria gozar de atenção e crédito.

Mais recentemente, uma pesquisa na base de dados PubMed revelou artigos que denunciam essas 
TABELA 1. Sites especializados em denúncias e esclarecimentos sobre terapias fraudulentas, 2011

\begin{tabular}{|c|c|}
\hline Nome & URL \\
\hline Quackwatch - Your Guide to Quackery, Health Fraud, and Intelligent Decisions & http://www.quackwatch.org \\
\hline National Council Against Health Fraud & http://www.ncahf.org/ \\
\hline The Australian Council Against Health Fraud Inc & http://www.acahf.org.au/ \\
\hline CSI - The Committee for Skeptical Inquiry & $\begin{array}{l}\text { http://www.csicop.org/ } \\
\text { http://www.skeptic.com/downloads/ }\end{array}$ \\
\hline Top Ten Things You Should Know About Alternative Medicine & Alternative_Medicine_by_Harriet_Hall.pdf \\
\hline $\begin{array}{l}\text { Science-Based Medicine - Exploring issues and controversies in the relationship } \\
\text { between science and medicine }\end{array}$ & http://www.sciencebasedmedicine.org/ \\
\hline Skeptical Information Links: Alternative Medicine & http://www.discord.org/skeptical/Alternative_Medicine/ \\
\hline $\begin{array}{l}\text { The James Randi Educational Foundation (JREF) - An educational resource } \\
\text { on the paranormal, pseudoscientific, and the supernatural }\end{array}$ & http://www.randi.org/site/ \\
\hline Museum of Questionable Medical Devices & http://www.museumofquackery.com/index2.htm \\
\hline Naturowatch - The Skeptical Guide to Naturopathic History, Theories, and Practices & http://www.naturowatch.org/ \\
\hline The Quack-Files & http://www.quackfiles.com/ \\
\hline Bay Area Skeptics - The San Francisco Bay Area's skeptical organization since 1982 & http://www.baskeptics.org/about \\
\hline ASR - Association for Science and Reason (Canada) & http://skepticscanada.org/ \\
\hline Cancer Treatment Watch - Your Guide to Intelligent Treatment & http://www.cancertreatmentwatch.org/ \\
\hline NTS - The North Texas Skeptics & http://www.ntskeptics.org/ \\
\hline Veterinarywatch & http://www.veterinarywatch.com \\
\hline Charlatanismo e Distrofia Muscular & http://www.distrofiamuscular.net/charlatanismo.htm \\
\hline The Skeptic's Dictionary - From Abracadabra to Zombies & http://www.skepdic.com \\
\hline PhACT — Philadelphia Association for Critical Thinking & http://www.phact.org/ \\
\hline Warning: Six Ways to Spot an Internet Marketing Charlatan & $\begin{array}{l}\text { http://www.leveltendesign.com/blog/colin/ } \\
\text { warning-six-ways-spot-internet-marketing-charlatan }\end{array}$ \\
\hline Skeptic News & http://www.skepticnews.com/ \\
\hline Internet Health Pilot - Your Gateway to Reliable Health Information & http://www.ihealthpilot.org/ \\
\hline
\end{tabular}

fraudes há mais de meio século (27). Em 1993, quando a Internet ainda era mal conhecida fora dos meios acadêmicos, o conselho holandês de saúde divulgou um relatório sobre o uso de métodos de cura alternati$\operatorname{vos}(26)$, concluindo que, mesmo ao final do século XX, a "cura pela fé" envolvia um crescente mercado de ilusões, tanto em número de consumidores como em variedade de produtos.

\section{MARKETING DA FRAUDE E SEUS CONSUMIDORES}

Os lucros auferidos pelas terapias desonestas são proporcionais ao rol de malefícios causados. Há ervas que, além de ineficazes no tratamento da AIDS, interferem no efeito dos inibidores de proteases usados no controle da doença (3). A Aaron Company, que comercializava ervas naturais que contêm efedrina, um estimulante do sistema nervoso central (também condenada como doping nas atividades esportivas de competição) (28), conseguiu um acordo judicial em um processo no qual era acusada de vender uma substância de prata (mild silver protein) capaz de controlar o $\mathrm{HIV}$, entre outras doenças (29). Apesar de ter tido sua comercialização proibida, o produto ainda é divulgado em sites, assim como os seus efeitos miraculosos $(30,31)$. Além disso, é possível adquirir um gerador Deluxe Colloidal Silver Generator (31) (US\$ 125 a 155 a unidade) - que permite ao consumidor produzir sua própria prata coloidal.

Também há casos de surpreendente estabilidade: encontra-se na web, há mais de uma década, propaganda de variados dispositivos (32-34) que emitem correntes elétricas capazes de exterminar em minutos os vírus e parasitas intestinais causadores de graves condições crônicas. A ação terapêutica alcançaria qualquer estágio da evolução de males como as fibromialgias, colites crônicas, dores articulares em geral, diabetes, glaucoma e qualquer tipo de câncer. Uma genuína panacéia moderna à venda nas livrarias (32) e à exposição na web (34). Tal caso é mais detalhadamente apresentado em um livro (32), que pondera sobre o desperdício de dias e semanas exigidos para a cura de afecções danosas que poderiam ser erradicadas em minutos por intermédio de dispositivos como syncrometers e zappers.

Existe, decerto, um crescente afluxo social na direção das informações sobre saúde na web - mídia de eleição porque mais influente (35) e globalizada, apesar das limitações de confiabilidade. Seja qual for a intencionalidade ou a forma de promoção dos divulgadores, cabe refletir sobre os mecanismos do marketing fraudulento em contato com a impressionante credulidade de seus clientes. Não obstante, reconhecida a dificuldade de tipificação dos terapeutas viciosos (35), sobretudo entre bilhões de sites, resta-nos o desafio de caracterizar e segmentar seus consumidores. Haveria como identificar e descrever as características do típico consumidor de fraudes?

Astin (36) tentou traçar contornos de uma segmentação naquilo que chamou de "preditores de recurso à assistência alternativa". Em seu estudo, os consumidores desses produtos tendiam a ter escolaridade mais alta. Não raro, associavam-se a grupos comprometidos com o ambientalismo e o feminismo. Explicitavam com frequência interesse em crescimento pessoal pela espiritualidade, que o autor chamou de "orientação holística à saúde". À primeira vista, tais 
categorizações podem parecer intuitivamente plausíveis, embora não plenamente esclarecedoras. Sabe-se de muitos notórios consumidores de terapias alternativas que não se enquadrariam nos perfis acima descritos. É difícil associar estadistas como Adolf Hitler, Stalin (37) e Ronald Reagan (38) a campanhas ambientalistas, feministas ou espiritualistas. Decerto, multiplicaram-se as "fachadas do eu" dos consumidores (39), tão ambivalentes e cambiantes quanto a nova mídia organizada em rede sobre a qual projetam suas predileções terapêuticas transitórias. Observa-se que o consumidor em saúde tornou-se um experimentador (40) que, sob vetores conflitantes, sobretudo sob o desgaste da dor crônica ou da iminência da morte, tornase facilmente incoerente. Observa-se que o mesmo indivíduo encarna variadas demandas, sem nítida uniformidade ou padrão de consumo tipificável (40). Em outros termos, há múltiplos consumidores em uma só pessoa, com demandas em profusão sobrepostas por vetores culturais e contingências do adoecer. $\mathrm{O}$ consumidor em tempos pós-modernos exibe predileções inconsistentes, ocupando espaços específicos em situações sociais influenciadas por singularidades, muito mais do que por generalizações (40). Suas predileções são estabelecidas por força das experiências pessoais e da oferta de muitas informações, filtradas segundo suas inclinações de momento, que não mais demandam uma referência única e fixa (41).

\section{CULTURA CONTEMPORÂNEA E MERCADO DA INSEGURANÇA}

A consulta ao Oxford textbook of public health (42) revela vários capítulos dedicados a crenças e valores culturais como definidores de doenças e condicionantes de saúde. Não é inédito nem surpreendente, portanto, que as crenças e os valores interfiram na percepção das ameaças à saúde sob as circunstâncias específicas aqui descritas. Ideias condicionam significações culturais das doenças, interferindo em sua prevalência, além de decidir sobre o sucesso ou incontrolabilidade de males que implicam incapacidade, adoecimento e morte. Caberia questionar, então, se um determinado conjunto de ideias (ou sua negação sistemática) poderia se transformar em gerador de vulnerabilidades e malefícios a incidir sobre uma sociedade estruturada a partir desse ideário. Em outros termos, será que a crença na proliferação das ameaças à saúde pública, na percepção de falibilidade das barreiras de proteção e lapsos dos sistemas de recuperação e no colapso das redes sociais de suporte poderiam criar condições para novos problemas de saúde pública? Caso a resposta seja positiva, como acreditamos ser, talvez seja infrutífero investir em intervenções de esclarecimento por evidências, de natureza informativa, visto que o esclarecimento racional no campo das panacéias tem se mostrado ineficaz quando em confronto com crenças, valores e expectativas atuais radicadas em medos ancestrais.

Um maior interesse cultural no cuidar de si resulta na intensificação dos acessos à grande rede, tanto como fonte de esclarecimento, mostruário de informações e produtos, como também como recurso extremo em contextos de desesperança. Os atributos de capilarização e os múltiplos formatos de sedução e engano (que ampliam o interesse pela nova mídia) se inclinam a favor da reprodução de textos falsamente esclarecedores. Frente a condições irrefreáveis, quando a medicina nos expõe à nossa própria finitude, parece essencial encontrar o amparo de outros sistemas de crenças à disposição nos templos ou no Google. Decerto, o crescimento exponencial de serviços e de informação multiplicou a variedade de meios e intenções de compra e venda. Nunca houve tantos sites e comunidades virtuais aos quais os portadores de condições crônico-degenerativas podem recorrer para encontrar acolhimento, informações técnicas, grupos de suporte (43) ou, até mesmo (nos casos mais controversos), autodiagnóstico (44). Por outro lado, também se descortinaram amplos espaços para conexão íntima entre pacientes vulnerabilizados por moléstias desgastantes - que não hesitam em recorrer a terapeutas nem sempre comprometidos com nobres ideais, por mais implausíveis que sejam seus discursos de sedução. Diversos estudos dão conta da avidez no consumo de informações sobre saúde, sobretudo no contexto de doenças incuráveis em estágio avançado (45). Segundo Eguchi et al., 32\% dos pacientes portadores de tumores malignos recorrem a terapias de eficácia não comprovada. Os autores acrescentam que, perante tais resultados, parecem existir lacunas de comunicação entre pacientes e seus médicos, sobretudo no que se refere às expectativas de cura oferecidas por essas modalidades de terapia (46). Os trabalhos clássicos no terreno dos cuidados paliativos descrevem uma terceira fase de adaptação aos prognósticos reservados, após as fases de "negação" e "fúria", na qual os pacientes se valem de táticas de barganha como forma de sobrevivência psíquica (47). Nesse ponto, os pacientes já admitem a inexorabilidade da doença e tentam desesperadamente se apegar a qualquer aceno de salvação. Mais frequentemente, as esperanças recaem sobre Deus e os sistemas terapêuticos metafísicos, não raro os que propõem processos de ruptura com a lógica da medicina convencional em vista da negociação de uma cura improvável. Kübler-Ross também afirma que o único sentimento que persiste por todas as cinco fases é a esperança, sempre presente em variadas formas. Mesmo os mais conformados e realistas estão sempre à procura de inovações terapêuticas, quaisquer que sejam suas origens ou formatos. Em condições de "dor total", como Saunders (48) denominou os pacientes em sofrimento físico, psíquico, social (pela sensação de isolamento, rejeição, dependência e inutilidade) e espiritual (pelo medo do pós-morte, das culpas perante Deus), instala-se a falta de discernimento, conduzindo a escolhas desesperadas que não se dariam em condições normais.

Portanto, torna-se necessário reconhecer que, nesse terreno, de nada valem as teorias clássicas do marketing acerca da segmentação e dos padrões fixos de consumo (49), que poderiam se prestar a substrato 
para campanhas de conscientização contra os terapeutas desonestos. O medo da dor, do envelhecimento e da morte - original, universal e inato, segundo Freud - nos conecta persistentemente à consciência de nossa inexorável degradação carnal. Acreditamos, portanto, que os modelos explicativos das leis do mercado livre - demanda e oferta - não esgotam totalmente a riqueza de interpretações nesse cenário. A lógica de mercado pela qual os consumidores devem ter acesso ao pleno esclarecimento para a seleção dos melhores produtos não se aplica à população vulnerabilizada por doenças graves, que busca restaurar uma vida sem sofrimento.

Em síntese, a revisão dos casos mais estáveis de panacéias diz algo sobre pelo menos dois aspectos relacionados à economia das ilusões na web. Primeiramente, parece-nos que qualquer tipo de artefato inócuo pode se tornar produto vendável, desde que se organize um arsenal de argumentos paracientíficos minimamente plausível que gere esperanças (50). O segundo ponto a destacar se refere às necessidades de um público consumidor temporariamente privado do juízo crítico por dor ou desespero. Tais consumidores se tornam insensíveis às ponderações que apelam ao senso médico e desconsideram a vulnerabilidade. Isso nos leva a advogar a capacitação das estruturas assistenciais existentes para fornecer suporte específico para pacientes portadores desse tipo de condição. Talvez a educação e a capacitação dos profissionais de saúde nesses temas nos conduzam a sucessos mais perenes do que os até agora conquistados pelas campanhas de informação pública. Além das tecnologias de diagnósticos e terapias, parece existir uma demanda por comunicação interpessoal (51) em situações de dúvida, medo e falta de recursos de informação e esperança, o que talvez favoreça o mercado das panacéias.

\section{CONCLUSÃO}

Em plena era do racionalismo e do desencantamento com as raízes dos processos de cura, os defensores dos direitos dos consumidores em saúde combatem a comercialização de artefatos produzidos sob sistemas metafísicos de argumentação falsa. Porém, analisar o fenômeno ignorando a vulnerabilidade ligada a condições física e emocionalmente desgastantes, sem considerar as demandas de integralidade e comunicação, pode levar aos mesmos resultados desapontadores. Deve-se reconhecer a configuração de um modelo de transação ideal, que reúne o desejo crucial de sobrevivência e a oferta ilimitada de esperanças na forma de panacéias, que suprem as perspectivas insatisfatórias demais para o gosto do ser humano contemporâneo na busca hedonista da saúde e da imortalidade do corpo. As novas panacéias compõem os lapsos deixados por séculos de racionalidade instrumental insensível à imaterialidade dos medos e desejos de sobrevivência humanos.

Agradecimento. Este trabalho foi financiado pelo Conselho Nacional de Pesquisa e Desenvolvimento Científico e Tecnológico - MCT/CNPq (edital no. 014/2008 - Universal).

\section{SYNOPSIS}

\section{Panaceas disseminated over the Internet and vulnerable patients: how to check a market of illusions?}

This article discusses the proliferation of medical quackery and fraud appearing and disappearing daily on the Internet. The customers of these scams, made vulnerable by disease or the prospect of death, use the Internet to buy products that would probably be ignored in other contexts. This vulnerability is linked to strenuous physical demands that compromise the ability to make decisions. The attempt to control the phenomenon of fraud as strictly rational, without taking into account the vulnerability of consumers who have little to lose and not considering their demands for comprehensive care, can lead to disappointing results, since these nostrums seem to be filling the gaps left by health care structures that have been insensitive to the immaterial nature of human fears.

Key words: internet; nostrums; quackery; health vulnerability; health communication.

\section{REFERÊNCIAS}

1. Food and Drug Administration. A study of health practices and opinions. Springfield: National Technical Information Service, U.S. Department of Commerce; 1972.

2. Green K. Marketing health care products on the Internet: a proposal for updated federal regulations. Am J Law Med. 1998;24(2-3):365-86.

3. Sullivan P. Internet a prime venue for snake-oil sales of "health products". CMAJ. 2001;165(4):465.

4. Bueno WC. Jornalismo científico, lobby e poder. Parc Estrat. 2001;13:168-200.
5. Ross K. Crackdown on unproven cancer treatments focuses on internet marketers. J Natl Cancer Inst. 2008;100(17): $1200-2$.

6. Barrett S, Jarvis WT. How quackery sells. Disponível em: http://www. quackwatch.org/01QuackeryRelated Topics/quacksell.html. Acessado em 21 de setembro de 2010.

7. Barrett SJ, Jarvis W. The health robbers: a close look at quackery in America. Amherst: Prometheus; 1993.

8. Porter R. A linguagem do charlatanismo na Inglaterra, 1660-1800. Em: Burke P,
Porter R, orgs. História social da linguagem. São Paulo: UNESP; 1997. Pp. 83-119.

9. Porter R. Quacks: fakers \& charlatans in medicine. London: Trafalgar Square; 2003.

10. Eisenberg DM, Kessler RC, Foster C, Norlock FE, Calkins DR, Delbanco TL. Unconventional medicine in the United States. Prevalence, costs, and patterns of use. N Engl J Med. 1993;328(4):246-52.

11. Barrett S. Quackery: how should it be defined? Disponível em: http://www. quackwatch.org/01QuackeryRelated 
Topics/quackdef.html. Acessado em 21 de setembro de 2010.

12. Lopes IL. Novos paradigmas para avaliação da qualidade da informação em saúde recuperada na web. Ci Inf. 2004; 33(1):81-90.

13. Health On the Net Foundation. The HON code of conduct for medical and health web sites. Disponível em: http:/ / www.hon.ch/HONcode/Conduct.html. Acessado em 21 de setembro de 2010.

14. The Education Wonks. Redefining parental rights: the latest battle. Disponível em: http://educationwonk. blogspot.com/2006/07/redefiningparental-rights-latest.html. Acessado em 21 de setembro de 2010.

15. ScienceBlogs. Update on Starchild Abraham Cherrix. Disponível em: http:// scienceblogs.com/insolence/2006/06/ update_on_abraham_starchild_ch.php. Acessado em 21 de setembro de 2010.

16. Bren L. Agencies team up in war against internet health fraud. Disponível em: http://www.fda.gov/Drugs/Emer gencyPreparedness/Bioterrorismand DrugPreparedness/ucm137264.htm. Acessado em 21 de setembro de 2010.

17. Federal Trade Commission. Operation Cure.All wages new battle in ongoing war against Internet health fraud. Disponível em: http://www.ftc.gov/ opa/2001/06/cureall.shtm. Acessado em 21 de setembro de 2010.

18. De Barros J. Dispensers, obeah and quackery: medical rivalries in postslavery British Guiana. Soc Hist Med. 2007;20(2):243-61.

19. Ndububa VI. Medical quackery in Nigeria: why the silence? Niger J Med. 2007;16(4):312-7.

20. Umre GA. Quackery - a burning threat of human health. J Indian Med Assoc. 2007;105(8):423.

21. Amin M, Hughes J, Timon C, Kinsella J. Quackery in head and neck cancer. Ir Med J. 2008;101(3):82-4.

22. Haycock DB, Wallis P. Quackery and commerce in seventeenth-century London: the proprietary medicine business of Anthony Daffy. Med Hist Suppl. 2005;25:1-216.

23. Sherman N. US agency warns on web herb claims. Disponível em: http:// www.asia.cmpmedica.com/ cmpmed ica_my/dispserchcontent.cfm?id=3947 \&image $=\operatorname{logo}$. gif $\& w=240 \& h=74$. Acessado em 21 de setembro de 2010.
24. Quackwatch - Your Guide to Quackery, Health Fraud, and Intelligent Decisions [site]. Disponível em: http:// www.quackwatch.org. Acessado em 21 de setembro de 2010.

25. Quackwatch. Recommended consumer protection publications. Disponível em: http://www.quackwatch.org/04Con sumerEducation/booklist.html. Acessado em 21 de setembro de 2010.

26. Huisman F. Shaping the medical market on the construction of quackery and folk medicine in Dutch historiography. Med Hist. 1999;43(3):359-75.

27. Fields O. Current campaigns against medical quackery. Fed Bull. 1952;38(1): 9-14.

28. Federal Trade Commission. Analysis of proposed consent order to aid public comment. Disponível em: http://www. $\mathrm{ftc}$.gov/os/2001/06/aaronanalysis.htm. Acessado em 21 de setembro de 2010.

29. Medicinal silver solutions. Lyme disease \& HIV. Disponível em: http:// www.xpressnet.com/bhealthy/bhealth y.html\#viri. Acessado em 21 de setembro de 2010.

30. Natural Health and Longevity Resource Center. Germ warfare against America: Part VIab - Methods of treatment of GWI. Disponível em: http://www.allnatural.com/part-6ab.html. Acessado em 21 de setembro de 2010.

31. Adachi K. The Deluxe colloidal silver generator. Disponível em: http://educate-yourself.org/cs/csgendesc.shtml. Acessado em 21 de setembro de 2010.

32. Clark HR. The cure for all diseases. San Diego: New Century Press; 1995.

33. Dr. Clark Information Center. Dr. Clark's discoveries and inventions. Disponível em: http://www.drclark. net/en/hulda_clark/discoveries.php. Acessado em 21 de setembro de 2010.

34. Dr. Clark Information Center [site] Disponível em: http:/ / www.drclark. net. Acessado em 21 de setembro de 2010.

35. Sillence E, Briggs P, Harris P, Fishwick L. Going online for health advice: changes in usage and trust practices over the last five years. Interact Comput. 2007;19(3):397-406.

36. Astin JA. Why patients use alternative medicine: results of a national study. JAMA. 1998;279(19):1548-53.

37. Gordon R. An alarming history of famous and difficult patients. New York: St. Martin's Press; 1997.
38. ParaScope. Bad times for Bonzo: star power is put to use at the White House. Disponível em: http:/ / www.parascope. com/articles / 0497/reagan01.htm. Acessado em 21 de setembro de 2010.

39. Goffman EA. Representação do eu na vida cotidiana. Petrópolis: Vozes; 2003.

40. Bauman Z. Vida para consumo. São Paulo: Jorge Zahar; 2007.

41. Bauman Z. Ética pós-moderna. São Paulo: Paulus; 1997.

42. Detels R, McEwen J, Beaglehole R, Tanaka H, eds. Oxford textbook of public health. New York: Oxford University Press; 2002.

43. Ziebland S, Chapple A, Dumelow C, Evans J, Prinjha S, Rozmovits L. How the internet affects patients' experience of cancer: a qualitative study. BMJ. 2004; 328(7439):564.

44. Vasconcellos-Silva PR, Castiel LD. As novas tecnologias de autocuidado e os riscos do autodiagnóstico pela Internet. Rev Panam Salud Publica. 2009;26(2): 172-5.

45. Cassileth BR, Brown H. Unorthodox cancer medicine. CA Cancer J Clin. 1988;38(3):176-86.

46. Eguchi K, Hyodo I, Saeki H. Current status of cancer patients' perception of alternative medicine in Japan. A preliminary cross-sectional survey. Support Care Cancer. 2000;8(1):28-32.

47. Kübler-Ross E. On death and dying: what the dying have to teach doctors, nurses, clergy and their own families. London: Routledge; 2009.

48. Saunders C. Hospice and palliative care: an interdisciplinary approach. London: Edward Arnold; 1991.

49. McCarthy J. Basic marketing. Homewood: Richard Irwin; 1960.

50. Wahlberg A. A quackery with a difference-new medical pluralism and the problem of 'dangerous practitioners' in the United Kingdom. Soc Sci Med. 2007;65(11):2307-16.

51. Andersson G, Carlbring P, Berger T, Almlov J, Cuijpers P. What makes internet therapy work? Cogn Behav Ther. 2009;12:1. [Epub ahead of print].

Manuscrito recebido em 27 de junho de 2010. Aceito em versão revisada em 23 de novembro de 2010. 